\title{
Nach der Therapie leidet die Lebensqualität
}

Viele Patienten mit lokal begrenztem Prostatakarzinom erleben, wenn sie sich einer radikalen Therapie unterziehen, Einschränkungen in Sexual-, Harnwegsund Darmfunktionen, die sich auch auf die Lebensqualität auswirken. Das zeigen die Berichte von 1.643 Patienten der ProtecT-Studie. 545 Patienten erhielten ein aktives Monitoring (regelmäßiger PSA-Test und klinische Untersuchung mit der Möglichkeit, bei Progression auf eine radikale Therapie zu wechseln), 553 eine radikale Prostatektomie und 545 eine Radiotherapie mit neoadjuvanter Androgendeprivation.

Die Prostatektomie wirkte sich von allen drei Therapiestrategien am negativsten auf Sexualfunktionen und Harnkontinenz aus, auch wenn sich ein Teil der Patienten im Laufe der Zeit davon erholte.

Die negativen Auswirkungen der Radiotherapie auf die Sexualfunktionen waren nach sechs Monaten am stärksten ausgeprägt, anschließend trat eine Erholung und Stabilisierung ein. Auf die Harnkontinenz hatte die Radiotherapie nur geringe Auswirkungen. Dagegen waren Harnverhalt und Nykturie nach sechs Monaten in der Radiotherapiegruppe am stärksten ausgeprägt, die anschließende Erholung führte nach zwölf Monaten zu ähnlichen Werten wie in den anderen Gruppen. In der Beobachtungsgruppe verschlechterten sich Sexual- und Harnwegsfunktionen nur graduell.

Was die Darmfunktion betraf, führte die Radiotherapie zu den schlechtesten

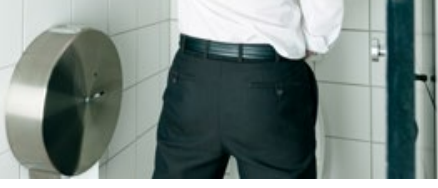

Prostatektomie und Radiotherapie können die Harnwegsfunktionen beeinträchtigen.

6-Monats-Befunden. Mit Ausnahme einer erhöhten Häufigkeit von Blutstühlen verbesserte sie sich dann jedoch wieder. In den anderen beiden Gruppen blieb die Darmfunktion weitgehend unverändert.

Die von den Patienten wahrgenommene Lebensqualität spiegelte die Veränderungen im Hinblick auf Sexual-, Darmund Harnwegsfunktionen wider.

Kathrin von Kieseritzky

Donovan JL et al. Patient-Reported Outcomes after Monitoring, Surgery, or Radiotherapy for Prostate Cancer. N Engl J Med. 2016;375(15):1425-37.

PD-1-Hemmer bei Kopf-Hals-Tumoren

\section{Längeres Leben nach Rezidiv}

Patienten mit platinrefraktärem Rezidiv eines Plattenepithelkarzinoms im KopfHals-Bereich profitierten in einer aktuellen Studie von einer Therapie mit dem PD-1-Hemmer Nivolumab. Relativ zur Standardtherapie war ihr Sterberisiko im Zeitraum von knapp 17 Monaten um $30 \%$ geringer. Absolut gesehen bedeutete das einen Zeitgewinn von im Median 2,4 Monaten.

Das zeigen die Ergebnisse einer randomisierten offenen Phase-III-Studie, an der 361 Patienten beteiligt waren. Dabei erhielt ein Teil der Patienten alle zwei Wochen eine Dosis Nivolumab, die übrigen bekamen eine Standardtherapie

\section{kurz notiert}

\section{Systemische Immunantwort wichtig im Kampf gegen Krebs}

Für eine effektive Antitumoraktivität scheint nicht nur eine lokale, sondern auch eine systemische Immunantwort erforderlich. Zu diesem Ergebnis kommen Wissenschaftler der Stanford University, CA/ USA. Ihre Erkenntnisse könnten erklären, warum nur einige Krebspatienten auf Immuntherapien ansprechen, andere dagegen nicht oder nur geringfügig davon profitieren. Die Forscher analysierten mithilfe der Massenzytometrie 40 Parameter in Millionen lebender Zellen aus verschiedenen Geweben im ganzen Körper von Mäusen mit triple-negativem Brustkrebs, die resistent gegen Checkpointinhibitoren waren. Durch einen zweigleisigen Ansatz konnten sie in diesen Zellen eine erfolgreiche Immunantwort stimulieren und den Tumor in den Tieren eliminieren. Hierfür verwendeten sie tumorbindende Antikörper und Substanzen, die dendritische Zellen aktivieren. Dadurch stieg die Anzahl der Makrophagen, dendritischen Zellen und T-Zellen im Tumor innerhalb von drei Tagen stark an. Die Unterdrückung des Tumors begann ab dem achten Tag. Die Immunzellproliferation war zu diesem Zeitpunkt allerdings beendet. Dies interpretieren die Forscher dahingehend, dass zwar die initiale Immunantwort primär im Tumor erfolgt, die Immunantwort in anderen Teilen des Körpers aber für das Aufrechterhalten der Immunabwehr verantwortlich ist [Spitzer MH et al. Cell. 2017;168(3):487-502].

Judith Neumaier mit Methotrexat, Docetaxel oder Cetuximab.

Im Median betrug die Überlebenszeit für Patienten der Nivolumab-Gruppe 7,5 Monate, verglichen mit 5,1 Monaten bei Standardtherapie. Die Differenz war signifikant (Hazard Ratio für die Mortalität 0,$70 ; \mathrm{p}=0,01$ ). Die Differenz für die Rate des 1-Jahres-Überlebens lag bei 19,4 Prozentpunkten (36,0 vs. 16,6\%) zugunsten von Nivolumab. Bis zur Progression vergingen unter Nivolumab median 2,0 und unter Standardbehandlung 2,3 Monate. Mindestens sechs progressionsfreie Monate schafften 19,7\% der mit Nivolumab und 9,9\% der standardmäßig Therapierten. Generell betrug die Ansprechrate mit Blick auf die RECIST-Kriterien $13,3 \%$ in der Nivolumab- und 5,8\% in der Standardtherapiegruppe.

Durch die Behandlung verursachte $\mathrm{Ne}$ benwirkungen vom Grad 3 oder 4 erlitten 13,1\% der Patienten unter Nivolumab und $35,1 \%$ der Patienten unter der Standardmedikation. In puncto Lebensqualität blieb die Situation unter Nivolumab stabil, während sie sich bei den Patienten mit üblicher Therapie verschlechterte.

Robert Bublak

Ferris RL et al. Nivolumab for Recurrent

Squamous-Cell Carcinoma of the Head and Neck. N Engl J Med. 2016;375(19):1856-67. 